\title{
Multimodal Imaging in Choroidal Metastasis
}

\author{
Gilda Cennamo $^{\text {a }}$ Daniela Montorio $^{\mathrm{b}}$ Marianna Carosielli ${ }^{\mathrm{b}}$ Mario R. Romano ${ }^{\mathrm{c}}$ \\ Giovanni Cennamob

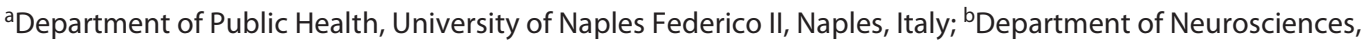 \\ Reproductive Sciences and Dentistry, University of Naples Federico II, Naples, Italy; 'Cye Unit, Humanitas-Gavazzeni \\ Hospital, Humanitas University, Bergamo, Italy
}

\section{Keywords}

Multimodal imaging - Enhanced depth imaging optical coherence tomography - Optical coherence tomography angiography $\cdot$ Choroidal metastasis

\begin{abstract}
Background: Choroidal metastasis represents the most common malignant intraocular tumours. Objectives: The objective of this study is to detect the structural and vascular features of choroidal metastasis by multimodal imaging. Methods: Sixteen eyes of 16 patients with choroidal metastasis were enrolled in this prospective study. The multimodal imaging was performed in all patients: fluorescein angiography, indocyanine green angiography, enhanced depth imaging optical coherence tomography (EDI-OCT), OCT angiography (OCTA), and ultrasonography. Results: The choroidal metastasis was located in the macula region in 9 eyes (57\%) and in the extramacular region in 7 eyes (43\%). EDIOCT showed a mean thickness of $950 \pm 246 \mu \mathrm{m}$, a smooth anterior tumour surface in 5 eyes (31\%), and a lumpy bumpy appearance in 11 eyes (69\%). The most frequent EDI-OCT features were represented by choriocapillaris thinning $(100 \%)$, shaggy photoreceptors (82\%), subretinal fluid with speckles (69\%), subretinal lipofuscin pigment (6\%), absence of drusen (100\%), optical shadowing (94\%), low-internal optical reflectivity (75\%), and retinal pigment epithelium alterations (43\%). OCTA revealed an absence of intratumoral vas-
\end{abstract}

cular network in all cases. Conclusions: The multimodal imaging contributed to greater insights into the anatomical and vascular features of choroidal metastasis. It allows for the collection of useful information to establish an appropriate diagnosis and follow-up.

(c) 2020 S. Karger AG, Basel

\section{Introduction}

Intraocular metastasis represents the most common malignant intraocular tumours, and they are often the first sign of dissemination of the primary tumour. The incidence of choroidal metastasis has increased over the years because of the improvement of diagnostic techniques and the increased survival of patients due to the improvement of available therapies [1-6].

Until a few years ago, choroidal metastasis was diagnosed not only by ultrasound but also by clinical exam and personal health history. Today, specific characteristics of choroidal metastases can be assessed by numerous other diagnostic procedures such as multicolour imaging, enhanced depth imaging optical coherence tomography (EDI-OCT), OCT angiography (OCTA), fluorescein angiography (FA), and indocyanine green angiography (ICGA) [7-17]. The aim of this prospective study was to use multimodal imaging to evaluate the vascular and morphological features of choroidal metastasis.

karger@karger.com

www.karger.com/ore

(c) 2020 S. Karger AG, Base

Karger!
Gilda Cennamo

Department of Neurosciences, Reproductive Sciences and Dentistry

Eye Clinic, University of Naples Federico II

Via S. Pansini 5, IT-80133 Naples (Italy)

xgilda@ hotmail.com 


\section{Materials and Methods}

In this prospective observational study, 18 patients with a diagnosis of choroidal metastasis were recruited from March 2014 to December 2018 at the Eye Clinic of the University of Naples "Federico II." They underwent complete ophthalmological examination including best-corrected visual acuity, ultrasound examination (Ascan standardized and B-scan), multicolour imaging, EDI-OCT, FA, ICGA, using spectral-domain (SD)-OCT (Spectralis, Heidelberg Engineering, Heidelberg, Germany, software version 1.10.2.0), and OCTA (AngioVue, RTVue XR Avanti, Optovue, Inc., Freemont, CA, USA). Only 10 patients underwent FA and ICGA.

The choroidal metastasis was evaluated for clinical features including the quadrantic location of the tumour epicentre, colour (pigmented, nonpigmented, or mixed), presence of orange pigment, overlying retinal pigment epithelium (RPE) alterations (none, atrophy, or hypertrophy), the presence of subretinal fluid, and lipofuscin pigment and drusen. Multicolour imaging, ultrasonography, and EDI-OCT documented these findings. The features recorded on A-scan and B-scan ultrasonography included the thickness (in millimetres), configuration (plateau, dome, or mushroom), echogenicity (solid or hollow), choroidal excavation (absent or present), and subretinal fluid (absent or present).

SD EDI-OCT was performed to detect the features of choroidal metastasis including the thinning of the choriocapillaris (absent or present), the contour of the anterior surface of the tumour (smooth or lumpy bumpy), the internal quality of the tumour, maximal tumour thickness (micrometres), and posterior choroidal shadowing (absent or present). Moreover, the intratumoral vascularization was evaluated by ICGA and OCTA. Patients were excluded if they had choroidal metastasis measuring more than $5 \mathrm{~mm}$ thickness by ultrasound, with the posterior margin of the lesions not visible on EDI-OCT. Further exclusion criteria included significant lens opacities and low-quality OCT and OCTA images.

\section{Optical Coherence Tomography Angiography}

OCTA images were obtained with the Optovue Angiovue System (software ReVue XR version 2017.1.0.151; Optovue Inc., Fremont, CA, USA), which is based on the split-spectrum amplitude decorrelation algorithm [18]. The images were captured with the standard angioretina protocol with a resolution of $6 \times 6 \mathrm{~mm}$ centred on the lesion. We manually segmented the choriocapillaris from $30 \mu \mathrm{m}$ below Bruch's membrane to $30-60 \mu \mathrm{m}$ below the RPE [15]. The images with a signal strength index less than 40 , residual motion artefacts, and incorrect segmentations were excluded from the analysis.

\section{Results}

During the enrolment period, 18 eyes of 18 patients with choroidal metastases that presented optimal quality of EDI-OCT and OCTA images were examined.
Table 1. Demographic, clinical, and ultrasonographic features of 16 eyes with choroidal metastasis

\begin{tabular}{|c|c|}
\hline & $N(\%)$ \\
\hline Eyes, $n$ & 16 \\
\hline Age mean (range), years & $58(37-78)$ \\
\hline \multicolumn{2}{|l|}{ Gender } \\
\hline Male & $5(31)$ \\
\hline Female & $11(69)$ \\
\hline \multicolumn{2}{|l|}{ Symptoms } \\
\hline None & $1(6)$ \\
\hline Present & $15(94)$ \\
\hline \multicolumn{2}{|c|}{ Primary site of the carcinoma } \\
\hline Breast & $10(63)$ \\
\hline Lung & $4(25)$ \\
\hline Pancreas & $1(6)$ \\
\hline Thyroid & $1(6)$ \\
\hline \multicolumn{2}{|l|}{ BCVA (Snellen) } \\
\hline$\geq 20 / 40$ & $6(38)$ \\
\hline$<20 / 40-20 / 100$ & $5(31)$ \\
\hline$<20 / 100$ & $5(31)$ \\
\hline \multicolumn{2}{|c|}{ Choroidal metastasis location } \\
\hline Macular & $9(57)$ \\
\hline Extramacular & $7(43)$ \\
\hline \multicolumn{2}{|c|}{ The mean thickness by echography } \\
\hline (median, range), $\mathrm{mm}$ & $2.8(2.85,1.0-3.6)$ \\
\hline \multicolumn{2}{|l|}{ Multicolour imaging } \\
\hline Yellow (Melanotic) & $2(12)$ \\
\hline Green (Amelanotic) & $14(88)$ \\
\hline Mixed & 0 \\
\hline \multicolumn{2}{|l|}{ Subretinal fluid } \\
\hline Present & $11(69)$ \\
\hline Absent & $5(31)$ \\
\hline \multicolumn{2}{|l|}{ RPE changes } \\
\hline None & $9(57)$ \\
\hline Atrophy & $4(25)$ \\
\hline Hyperplasia & $3(18)$ \\
\hline \multicolumn{2}{|l|}{ Orange pigment } \\
\hline Present & $1(6)$ \\
\hline Absent & $15(94)$ \\
\hline \multicolumn{2}{|l|}{ Drusen } \\
\hline Present & 0 \\
\hline Absent & $16(100)$ \\
\hline \multicolumn{2}{|l|}{ Echography configuration } \\
\hline Plateau & $3(18)$ \\
\hline Dome & $13(82)$ \\
\hline Mushroom & \\
\hline Echography hollowness & $6(38)$ \\
\hline
\end{tabular}

BCVA, best-corrected visual acuity; RPE, retinal pigment epithelium.

Two eyes were excluded because of low-quality of EDIOCT and OCTA images due to the peripheral localization of the tumour (at or anterior to the equator), thick tumours ( $>5 \mathrm{~mm}$ thickness in ultrasound examina- 
Table 2. EDI-OCT and OCTA features of 16 eyes with choroidal metastasis

$N(\%)$

\begin{tabular}{|c|c|}
\hline Tumour thickness, mean (median, range) $\mu \mathrm{m}$ & $11(69)$ \\
\hline \multicolumn{2}{|l|}{ Configuration } \\
\hline Dome & $4(25)$ \\
\hline Plateau & $12(75)$ \\
\hline \multicolumn{2}{|l|}{ Anterior tumour surface } \\
\hline Lumpy bumby & $11(69)$ \\
\hline Smooth & $5(31)$ \\
\hline \multicolumn{2}{|l|}{ Optical shadowing } \\
\hline Present & $15(94)$ \\
\hline Absent & $1(6)$ \\
\hline \multicolumn{2}{|l|}{ Optical reflectivity } \\
\hline Low & $12(75)$ \\
\hline High & $4(25)$ \\
\hline \multicolumn{2}{|l|}{ Associated features } \\
\hline Choriocapillaris thinning & $16(100)$ \\
\hline Shaggy photoreceptors & $13(82)$ \\
\hline Subretinal fluid with speckles & $11(69)$ \\
\hline Subretinal lipofuscin deposition (orange pigment) & $1(6)$ \\
\hline \multicolumn{2}{|l|}{ Associated retinal features } \\
\hline Bruch membrane intact & $16(100)$ \\
\hline RPE atrophy & $4(25)$ \\
\hline RPE hyperplasia & $3(18)$ \\
\hline Drusen absent & $16(100)$ \\
\hline Ellipsoid portion of photoreceptors alterations & $9(57)$ \\
\hline External limiting membrane alterations & $7(43)$ \\
\hline Outer nuclear layer alterations & $3(18)$ \\
\hline Outer plexiform layer alterations & $3(18)$ \\
\hline Inner retinal layers alterations & $2(12)$ \\
\hline \multicolumn{2}{|l|}{ OCTA findings } \\
\hline Absence of intratumoral vascular network & $16(100)$ \\
\hline
\end{tabular}

RPE, retinal pigment epithelium; EDI-OCT, enhanced depth imaging optical coherence tomography; OCTA, OCT angiography.

tion), significant lens opacities, and lack of patient collaboration.

A total of 16 eyes of 16 patients ( 11 females and 5 males; mean age $58 \pm 11$ years) were included in the study. Fifteen eyes (94\%) presented ocular symptoms and 1 patient $(6 \%)$ presented none. The primary site of the carcinoma was located in the breast $(n=10,63 \%)$ lung $(n=4$, $25 \%)$, pancreas $(n=1,6 \%)$, and thyroid gland $(n=1,6 \%)$. Twelve eyes $(75 \%)$ of patients were usually aware of the presence history of tumour. The location of the choroidal metastasis was represented by the macula in 9 eyes (57\%) and the extramacula in 7 eyes (43\%). Clinical features included the subretinal fluid in 11 eyes (69\%), RPE atrophy in 4 eyes (25\%), RPE hyperplasia in 3 eyes (18\%), orange pigment in 1 eye $(6 \%)$, and absence of drusen in all cases.
The mean thickness of choroidal metastasis measured by echography was $2.8 \mathrm{~mm}$ (range, $1.0-3.6 \mathrm{~mm}$ ). At multicolour images, the choroidal metastasis appeared green (amelanotic) in 14 eyes (88\%) and yellow (melanotic) in 2 eyes (12\%) (Table 1$)$.

The features of the choroidal metastasis recorded by EDI-OCT and OCTA are displayed in Table 2. The choroidal metastasis, measured by EDI-OCT, presented an average thickness of $950 \pm 246 \mu \mathrm{m}$ (median, $851 \mu \mathrm{m}$; range, $560-1,522 \mu \mathrm{m}$ ).

The EDI-OCT characteristics of choroidal metastasis were represented by the tumour configuration that was dome in 5 eyes (31\%) and plateau in 11 eyes $(69 \%)$. The lumpy bumpy $(n=11,69 \%)$ and the smooth $(n=5,31 \%)$ represented anterior tumour surface appearance (Fig. 1, 2). Optical shadowing was present in 15 eyes (94\%).

Other EDI-OCT features were choriocapillaris thinning (100\%), shaggy photoreceptors (82\%), and subretinal fluid with speckles (69\%) (Fig. 3). In all cases, there were no modifications in the inner retinal layers. OCTA displayed an absence of intratumoral vascular network in all 16 eyes.

\section{Discussion}

To our knowledge, this is the first prospective study to evaluate multimodal imaging in choroidal metastasis. The ultrasound represented the gold standard in the diagnosis of these lesions that appear as flat or slightly dome-shaped masses, with a medium-high inhomogeneous reflectivity, unlike the choroidal melanomas that are more frequently dome-shaped, with a homogeneous medium-low reflectivity. At ultrasound A-scan in choroidal metastases, the echoes had a moderate amplitude when compared to the echoes of melanomas that have a high-initial amplitude and a low-internal amplitude [19].

The use of the ultrasound presents several limitations, including the low likelihood of detecting very small lesions. Usually, tumours that cause a retinal lift greater than $1 \mathrm{~mm}$ can be demonstrated on ultrasound. When the tumour is visible and the ultrasound can be performed under optimal conditions, lesions that cause a lift of 0.5 $\mathrm{mm}$ can be detected. Moreover, smaller tumours can escape detection by ultrasound. However, the acoustic profile does not make it possible to differentiate the cytological characteristics of the lesions completely. The acoustic profile can be masked by structures that precede the tumour or by an incorrect alignment of the probe to the vitreous-tumour interface [20]. 
Fig. 1. The left eye of a patient (female, 67 years) affected by choroidal metastasis. a Multicolour imaging shows a yellow lesion, superotemporal to the fovea. b Fundus autofluorescence reveals an area of hyper/hypoautofluorescence. c EDI-OCT shows the typical "lumpy bumpy" anterior tumour surface. d OCTA of choroidal metastasis does not show any vascular network inside the lesion. EDI-OCT, enhanced depth imaging optical coherence tomography; OCTA, OCT angiography.

Fig. 2. The right eye of a patient (female, 65 years) affected by choroidal metastasis. a Multicolour imaging shows a green lesion, temporal to the fovea. b Fundus autofluorescence reveals an area of hyper/hypoautofluorescence. c EDI-OCT, the choroidal metastasis shows a smooth anterior tumour surface. d OCTA of choroidal metastasis does not show any vascular network inside the lesion. EDI-OCT, enhanced depth imaging optical coherence tomography; OCTA, OCT angiography.
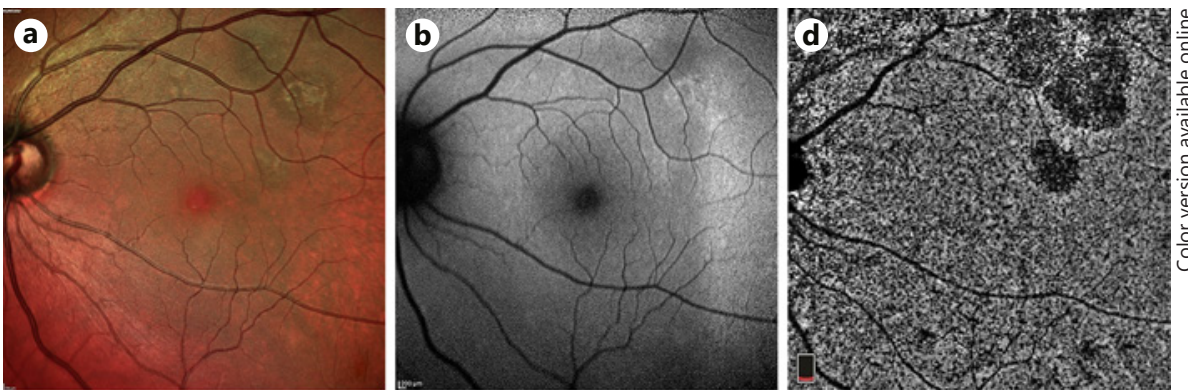

C
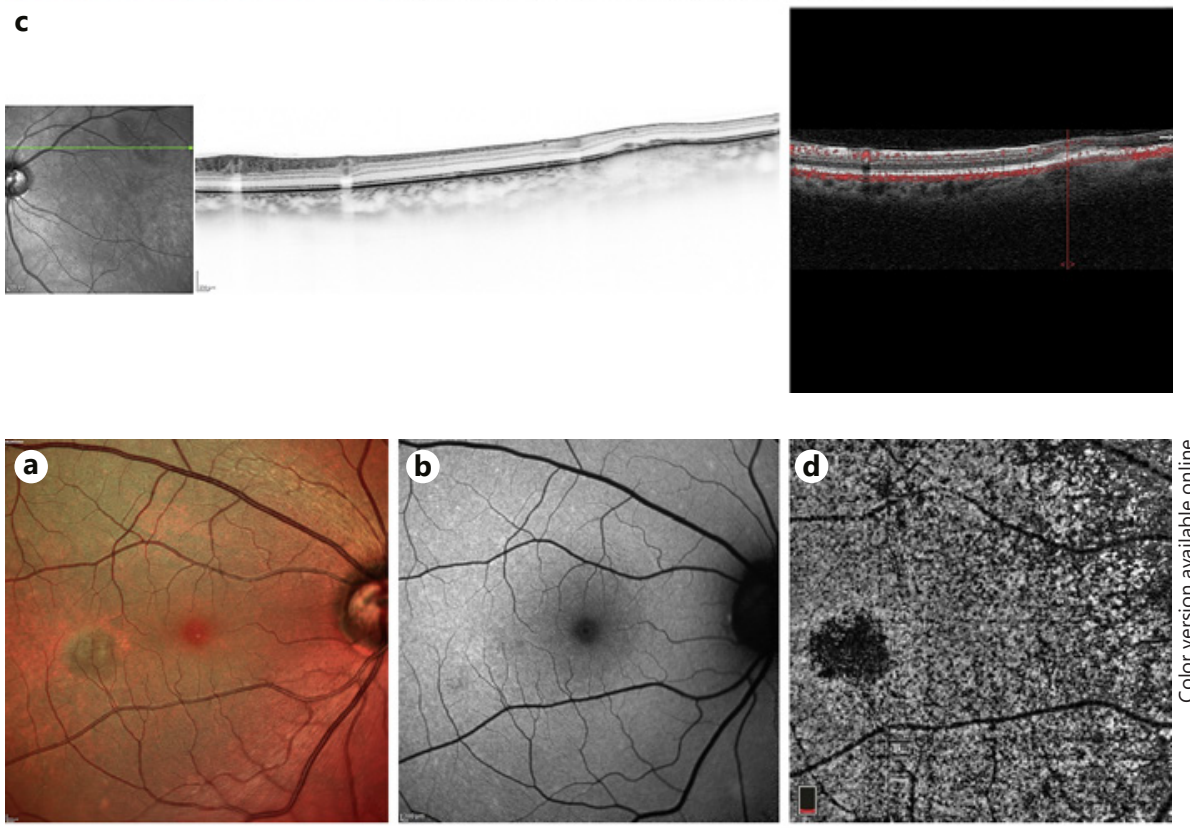

C
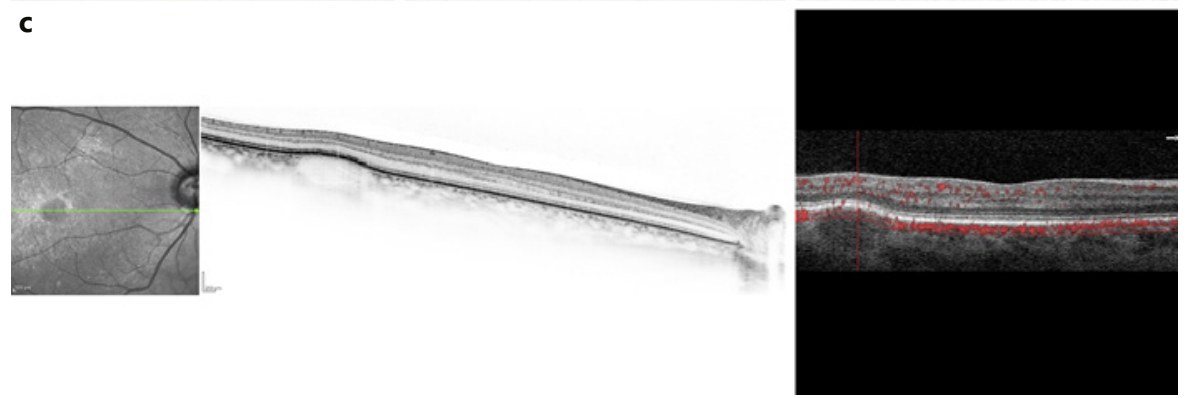

Typically, the choroidal metastases appear flat or plateau shaped, often associated with serous retinal detachment [7]. Very rarely, metastases may rupture the Bruch membrane and develop a mushroom shape [21].

The introduction of the multimodal imaging was useful in providing appropriate diagnosis and the management of the choroidal metastasis. The role of these techniques has become crucial to identify the anatomical and vascular features of these choroidal lesions and to establish their aetiology. The potential utility of multimodal imaging is not limited to diagnosis because it can also be beneficial in predicting the clinical course of these lesions.

At multicolour imaging, the choroidal metastases generally appear green because they are not pigmented while infrequently they can be pigmented and appear yellow [11]. The use of the EDI-OCT, a technique of the SDOCT introduced by Spaide and collaborators, allowed us to increase the resolution of the deeper layers of the choroid and the sclera and to identify better the morphological features of the choroidal metastasis [22]. 

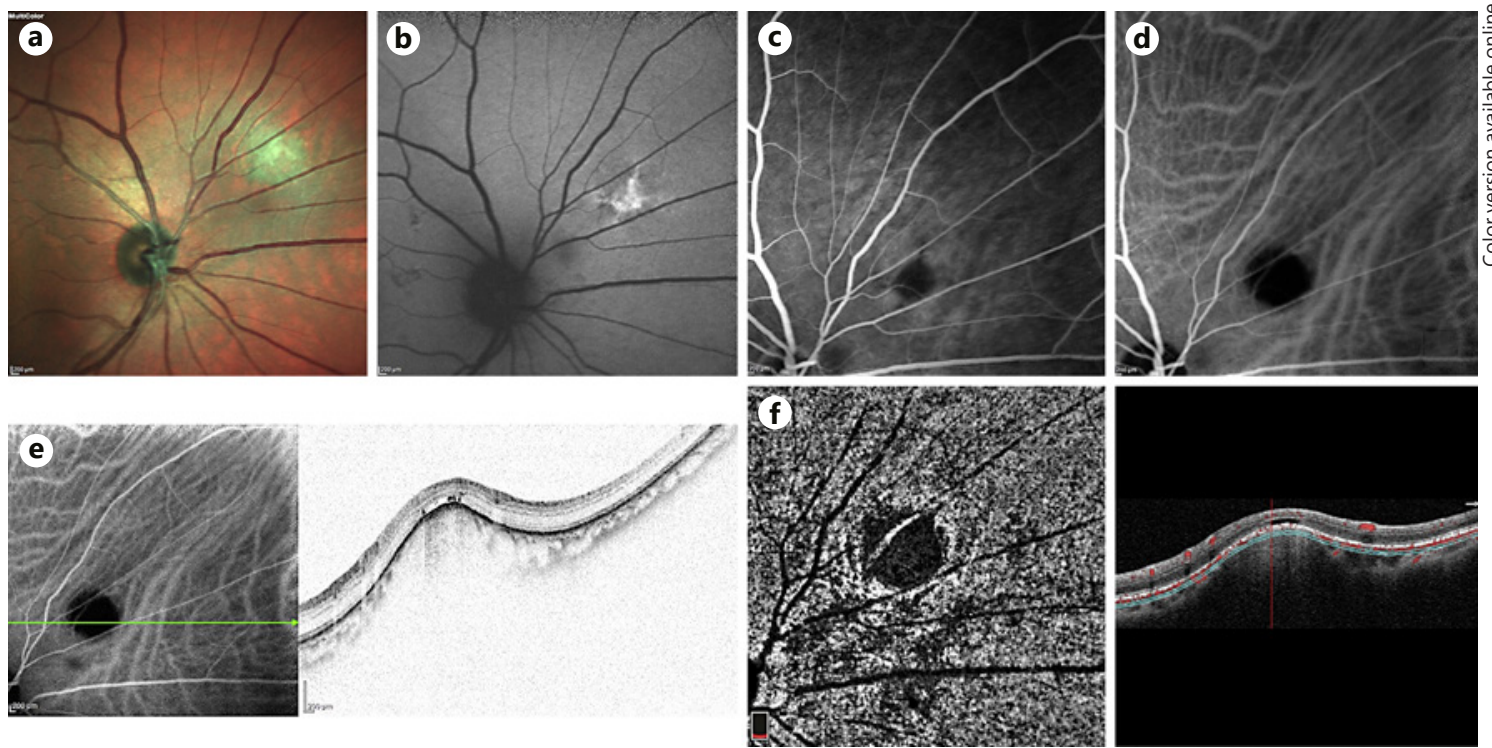

Fig. 3. The left eye of a patient (male, 60 years) affected by choroidal metastasis. a Multicolour imaging shows a green lesion, superonasal to the optic disk. b Fundus autofluorescence reveals an area of hyper/hypoautofluorescence. c, d. On FA and ICGA, the lesion appears hypofluorescent and hypocyanescent. e On EDI-OCT, the choroidal metastasis presents a low-internal reflectivity with the subretinal fluid and hyperreflective dots inside, known as "speckles". f OCTA of choroidal metastasis does not show any vascular network inside the lesion. EDIOCT, enhanced depth imaging optical coherence tomography; OCTA, OCT angiography; FA, fluorescein angiography; ICGA, indocyanine green angiography.

The main features of these lesions at the EDI-OCT are represented by the irregularity of the anterior surface of the tumour (lumpy bumpy), in contrast to the smooth appearance that appears on the ultrasound; the elongation and swelling of photoreceptors (shaggy photoreceptors); the subretinal fluid often with hyperreflective dots inside, known as "speckles," which may derive from the outer segment of photoreceptors [11,13]. The subretinal fluid density can be measured at OCT and appears significantly lower in choroidal metastases than in melanomas.

Other signs, detected by EDI-OCT, are the low-internal tumour reflectivity with enlargement of the suprachoroidal space (internal reflectivity may increase after treatment, due to an increase in the fibrotic component), the thinning of the choriocapillaris above the tumour and the partial masking of the choroid by the tumour, the modifications of the EPR (atrophy or hyperplasia), the loss of external limiting membrane and the external segmentinternal segment, and the irregularity of the internal plexiform layer $[7,11,13]$. Compared to ultrasound, EDIOCT was more sensitive in the evaluation of small tumours both at the time of presentation and after treatment $[11,23]$.

SD-OCT and OCTA in Choroidal Metastasis
Regarding the conventional imaging technique, the FA is not decisive for the diagnosis of choroidal metastases, because most choroidal tumours have the same angiographic features. Generally, hypofluorescence is observed in the early stages and a non-homogeneous hyperfluorescence in the late phases, while sometimes small hyperfluorescent points and areas of late leakage on the mass are observed [24]. Unlike FA, the ICGA is useful in the differential diagnosis of choroidal metastases, which usually appear hypofluorescent at all stages [25].

The "double circulation" pattern, described for the first time by Augsburger in choroidal melanomas, is rarely present in choroidal metastases. Tumour vessels are distinguishable from normal choroidal vessels by their random course [26].

ICGA is also useful because it shows an area of choroidal involvement by the broader metastasis than can be observed at the clinical examination or the FA [7]. The introduction of OCTA can help in the differential diagnosis, in a non-invasive way, of the various types of choroidal lesions. OCTA shows a lack of flow at the level of the choroidal metastases and absence of pathological blood flow at the level of the external retinal layers, while in melanomas, haemangioma, and choroid osteomas, 
there can be a dense and irregular vascular network within the tumour and a possible increase in flow in the external nuclear layer $[15,16]$.

In conclusion, the use of multimodal imaging, together with OCTA, contributed to greater insights into the anatomical and vascular features of the choroidal metastasis to distinguish them from other choroidal lesions. The combination of these techniques allowed us to collect useful information that shed light on the different pathogenesis of choroidal tumours and to predict the clinical course of these lesions over time.

\section{Statement of Ethics}

This study was approved by the Institutional Review Board of the University of Naples "Federico II" and all investigations adhered to the tenets of the Declaration of Helsinki. Written informed consents were obtained from the patients enrolled in this study.

\section{Conflict of Interest Statement}

The authors have no conflicts of interest to declare.

\section{Funding Sources}

The authors received no funding for this study.

\section{Author Contributions}

Gilda Cennamo, Mario R. Romano, and Giovanni Cennamo designed and directed this study. Gilda Cennamo, Daniela Montorio, and Marianna Carosielli collected and analysed the data, edited the manuscript. All authors discussed the results and commented on the manuscript.

\section{References}

1 Bloch RS, Gartner S. The incidence of ocular metastatic carcinoma. Arch Ophthalmol. 1971;85(6):673-5.

2 Eliassi-Rad B, Albert DM, Green WR. Frequency of ocular metastases in patients dying of cancer in eye bank populations. Br J Ophthalmol. 1996;80(2):125-8.

$3 \mathrm{Su} \mathrm{H-T}$, Chen Y-M, Perng R-P. Symptomatic ocular metastases in lung cancer. Respirology. 2008;13(2):303-5.

4 Merrill CF, Kaufman DI, Dimitrov NV. Breast cancer metastatic to the eye is a common entity. Cancer. 1991;68(3):623-7.

5 Freedman MI, Folk JC. Metastatic tumors to the eye and orbit. Patient survival and clinical characteristics. Arch Ophthalmol. 1987; 105(9):1215-9.

6 Nabi G, Dadeya S, Dogra PN, Lal H. Eye metastasis form urothelial tumours. Int Urol Nephrol. 2002;34(1):51-4.

7 Mathis T, Jardel P, Loria O, Delaunay B, Nguyen AM, Lanza F, et al. New concepts in the diagnosis and management of choroidal metastases. Prog Retin Eye Res. 2019;68:14476.

8 Vishnevskia-Dai V, Zur D, Yaacobi S, Moroz I, Newman H, Neudorfer M. Optical coherence tomography. An adjunctive tool for differentiating between choroidal melanoma and metastasis. J Ophthalmol. 2016;2016: 9803547.

9 Konstantinidis L, Damato B. Intraocular metastases: a review. Asia Pac J Ophthalmol. 2017;6(2):208-14.

10 Ishida T, Morohoshi K, Takeuchi Y, Soma R, Uchida M, Ohno-Matsui K. Swept-source op- tical coherence tomographic ndings in eyes with metastatic choroidal tumor. Am Journal Ophthalmol Case Rep. 2017;8:44-7.

11 Al-Dahmash SA, Shields CL, Kaliki S, Johnson T, Shields JA. Enhanced depth imaging optical coherence tomography of choroidal metastasis in 14 eyes. Retina. 2014;34(8):1588-93.

12 Augsburger JJ, Golden MI, Shields JA. Fluorescein angiography of choroidal malignant melanomas with retinal invasion. Retina. 1984;4(4):232-41.

13 Demirci H, Cullen A, Sundstrom JM. Enhanced depth imaging optical coherence tomography of choroidal metastasis. Retina. 2014;34(7):1354-9.

14 Arevalo JF, Fernandez CF, Garcia RA. Optical coherence tomography characteristics of choroidal metastasis. Ophthalmology. 2005; 112(9):1612-9.

15 Cennamo G, Romano MR, Breve MA, Velotti N, Reibaldi M, de Crecchio G, et al. Evaluation of choroidal tumors with optical coherence tomography: enhanced depth imaging and OCT-angiography features. Eye. 2017; 31(6):906-15.

16 Toledo JJ, Asencio M, García JR, Morales LA, Tomkinson C, Cajigal C. OCT Angiography: imaging of choroidal and retinal tumors. Ophthalmol Retina. 2018;2(6):613-22.

17 Natesh S, Chin KJ, Finger PT. Choroidal metastases fundus autofluorescence imaging: correlation to clinical, OCT, and fluorescein angiographic findings. Ophthalmic Surg Lasers Imaging. 2010;41(4):406.

18 Jia Y, Tan O, Tokayer J, Potsaid B, Wang Y, Liu JJ, et al. Split spectrum amplitude-decor- relation angiography with optical coherence tomography. Opt Express. 2012;20:4710-25.

19 Sobottka B, Schlote T, Krumpaszky HG, Kreissig I. Choroidal metastases and choroidal melanomas: comparison of ultrasonographic findings. Br J Ophthalmol. 1998;82(2):15961.

20 Coleman DJ, Abramson DH, Jack RL, Franzen LA. Ultrasonic diagnosis of tumors of the choroid. Arch Ophthalmol. 1974;91(5):34454.

21 Ward SD, Byrne BJ, Kincaid MC, Mann ES Ultrasonographic evidence of a mushroomshaped choroidal metastasis. Am J Ophthalmol. 2000;130(5):681-2.

22 Margolis R, Spaide RF. A pilot study of enhanced depth imaging optical coherence tomography of the choroid in normal eyes. Am J Ophthalmol. 2009;147(5):811-5.

23 Torres VLL, Brugnoni N, Kaiser PK, Singh AD. Optical coherence tomography enhanced depth imaging of choroidal tumors. Am J Ophthalmol. 2011;151(4):586-e2.

24 Meyer K, Augsburger JJ. Independent diagnostic value of fluorescein angiography in the evaluation of intraocular tumors. Graefes Arch Clin Exp Ophthalmol. 1999;237(6):48994.

25 Shields CL, Shields JA, De Potter P. Patterns of indocyanine green videoangiography of choroidal tumours. Br J Ophthalmol. 1995; 79(3):237-45.

26 Augsburger JJ, Golden MI, Shields JA. Fluorescein angiography of choroidal malignant melanomas with retinal invasion. Retina. 1984;4(4):232-41. 